\title{
Considerations about the present and future use of autoradiography
}

W.E. Stumpf

\section{Correspondence}

W.E. Stumpf

International Institute of Drug Distribution,

Cytopharmacology and Cytotoxicology

2612 Damascus Church Road

Chapel Hill, NC 27516

USA

E-mail: stumpfwe@email.unc.edu

Presented at the 5th International Symposium on Radioautography,

São Paulo, SP, Brasil,

August 24-26, 1997
University of North Carolina at Chapel Hill and International Institute of Drug Distribution, Cytopharmacology and Cytotoxicology, Chapel Hill, NC, USA
This Vth International Conference on Radioautography at the University of São Paulo follows the footsteps of earlier important contributions in this field by the present rector magnificus, Dr. Fava de Moraes, and the former chairman of the Department of Histology and Embryology and Dean of the Biomedical Sciences Institute, Dr. Carneiro. Thus, this conference is standing on traditional grounds. This conference provided an opportunity for the assessment of progress in the field of tissue autoradiography and its present potential as a tool in biomedical research at the University of São Paulo and other universities of Brazil, as well as at the international level.

Although this international conference was numbered as "the Fifth", it needs to be pointed out that several previous conferences on autoradiography or radioautography remained unnumbered and that the true number would most likely amount to ten or more.

During the conference both terms "Radioautography" and "Autoradiography" have been used. While correct and uniform terminology should be observed and discussions about terminology generally encouraged, this specific topic has been addressed repeatedly in the literature. Since 1973, the U.S. National Library of Medicine decided in favor of "autoradiography", which is the official singular subject heading for MEDLINE searches. A continued discussion of the semantics of these two terms thus is likely not fruitful. Both are linguistic hybrids of Latin and Greek. Their individual meaning is closely enough related to permit their parallel use without causing confusion. However, settlement for one term ultimately is to be advised.

While not reviewing individual papers of this conference, notable were those presentations that applied autoradiography a) as a complementary approach, that is, in conjunction with alternate procedures (e.g., immunohistochemistry, biochemistry), b) as carefully controlled quantitative assessment of synthetic cellular processes (e.g., DNA, protein), and c) for providing maps - especially for the brain and other highly complex organs - as a guide for follow-up studies toward the understanding of mechanisms of action of autacoids and xenobiotics.

Furthermore, it was apparent that autoradiographic studies, since generally more laborious and less expedient than biochemical studies, need to be planned with special care in terms of their experimental hypothesis and the significance of contributions of the expected results. Long exposure times must be considered and repeat, control and additional studies initiated in due time, often before the results of the experiment are known. 
Since the inception of autoradiography, which can now be viewed as a methodology, about 50 years ago, different autoradiographic techniques evolved. Through continued improvement and extended application, important discoveries have been made that contributed to the advancement of our knowledge, seminal in the development of new paradigms. With this histochemical method, cellular and subcellular processes can be studied in relatively intact tissue with topographical relationships maintained, thus providing information that is difficult or impossible to obtain by disruptive biochemical procedures. The use of radiolabeled compounds with high specific activity together with special photographic emulsion and the exposure-related signal amplification allows high sensitivity, another characteristic of autoradiography, in addition to the contextual tissue and cellular resolution.

Among the various autoradiographic techniques that have evolved, the following basic approaches can be distinguished:

- Liquid nuclear emulsion coating of specimen

- with glass-mounted untreated sections

- with glass-mounted treated sections, e.g., as for in situ hybridization, and for radiolabeled antibodies

- Dry emulsion apposition to tape-mounted dry specimen

- Dry emulsion frozen section thaw- or dry-mounting

- Dry emulsion apposition to in vitro incubated sections

When applications are considered, the various autoradiographic techniques may be categorized as:

- Whole body film or imaging plate autoradiography

- Precursor incorporation autoradiography

- Dry-mount and thaw-mount in vivo ligand receptor autoradiography

- In vitro section incubation receptor autoradiography

- Metabolic indicator (2-deoxyglucose) low resolution and high resolution autoradiography

- Combined autoradiography-immunohistochemistry

- Correlative (computer-imaging) autoradiography

- Electron microscope (transmission or freeze fracture) autoradiography

- Neutron activation autoradiography

- Photoaffinity labeling autoradiography

These techniques evolved at different times throughout the history of autoradiography and their range of application has been expanded.

A screening of individual investigators and the techniques used by them reveals that scientists who apply autoradiography effectively are highly specialized, using only one kind of technique. This amazing fact reflects an apparent need for such specialization, not merely regarding equipment, but predominantly for the acquisition of skills and experience for experimental design, execution, and interpretation of data. It also indicates that a casual or occasional use of autoradiography by biochemists, physiologists, or even histologists, not experienced in that specific area, may not obtain valid results, unless considerable effort is made and adequate experience is gained. Advice and guidance from, or collaboration with, someone experienced in the particular autoradiographic technique selected may need to be 
sought. In the past, such requirements have not been sufficiently recognized. As a consequence, investigators not experienced in autoradiography were sometimes mislead to publish artifacts as results.

As to the future of autoradiography, predictions suggest that there will be a continued need for studies with radiolabeled tracers. The techniques considered for further development and increased application include: correlative computer imaging and computer assisted quantification, combined autoradiography-immunohistochemistry, imaging plate analysis with a dry detection medium, in situ hybridization autoradiography, and in vivo and in vitro receptor-ligand autoradiography. Precursor incorporation autoradiography will remain in demand.

Electron microscope autoradiography deserves further attention. Improvements need to be aimed at higher expediency in order to make electron microscope autoradiography more applicable in molecular biology. A suitable electron microscopic autoradiography technique for the study of non-covalently bound diffusible compounds needs to be developed. Despite efforts and claims made in the literature, no such technique is available at present. Introduction and acceptance of an electron microscopic technique for diffusible compounds will be possible only after rigorous testing with two diffusible compounds known to be localized, and correct localization is demonstrated at both the light and electron microscopic levels with the same compounds and preparation techniques.

Improvements toward expediency of the use of autoradiography will be achieved through the development of radiation detection medium that does not require wet processing as is needed for the photographic image. However, in order to replace photographic emulsion with a dry detection medium, the high sensitivity and resolution of the photographic process need to be matched. This appears to be difficult and remained an obstacle in the past. Efforts to replace the use of radioisotopes as a marker also must be made. Success of such efforts would eliminate autoradiography. Ideally, the unmolested tissue is studied in vivo, as much as possible. Since information on specific tissue, cellular and subcellular levels of binding and deposition are required for the understanding of sites and mechanisms of action of bioactive compounds, the need to apply and further improve tracer technologies is apparent. Therefore, autoradiography will remain a valuable tool in modern biotechnology. 